\title{
Penetrating Keratoplasty with a Preserved Donor Cornea in Veterinary Ophthalmology
}

\author{
Boris. V. Usha ${ }^{1}$, Svetlana Y. Kontsevaya ${ }^{1}$, Vladimir I. Lutsay ${ }^{1}$, Inga. M. Nityaga ${ }^{1} \&$ Uliyana E. Lukashina $^{2}$ \\ ${ }^{1}$ Moscow State University of Food Production, Moscow, Russia \\ ${ }^{2}$ Shilkin Center of Veterinary Ophthalmology, Moscow, Russia \\ Correspondence: Boris. V. Usha, Moscow State University of Food Production, 125080, Moscow, Volokolamskoe \\ avenue, 11, Russia.E-mail: inga99@mail.ru
}

Received: September 6, 2019

Accepted: September 26, 2019

Online Published: October 17, 2019

doi:10.5539/jmbr.v9n1p71

URL: https://doi.org/10.5539/jmbr.v9n1p71

\begin{abstract}
The present article describes the penetrating keratoplasty using the technique of rehydration of donor cornea dehydrated over silica gel, as well as complications that can arise. The indications included corneal ulcers, descemetocoele, corneal sequestrum and corneal perforation. 37 (88.37\%) out of 43 corneal transplants had a favorable outcome in surgeries with complete excision of abnormal tissues. In 5 cases $(11.63 \%)$, the authors faced such complications as suture failure, formation of anterior synechias, incomplete epithelization after the suture removal, and transplant swelling reversed with the preservation of eye optical functions, and secondary glaucoma in the late postoperative period that ended up in eyeball endoprosthesis transplantation. Due to a high rate of efficiency of the proposed method, penetrating keratoplasty with rehydrated grafts can be recommended for the treatment in case of medical emergency experienced by cats and dogs.
\end{abstract}

Keywords: Transplant, Penetrating Keratoplasty, Donor Cornea, Recipient, Rehydration

\section{Introduction}

Severe corneal defects connected with loss of stromal tissue, corneal thinning and perforation, are a serious threat to the vision and always require emergency surgery. Indications include injuries, postinfective diseases such as corneal ulceration or malacia, as well as deep keratectomy during surgery. Surgical treatment is aimed at restoring the thickness of the cornea with the maximum possible preservation of eye visual functions, which is almost impossible during standard autoconjunctival plasty. This provides the rationale for the present research (Brunette, Rosolen, \& Carrier, 2011; Gelatt, Gilger, \& Kern, 2013; Pumphrey, Pizzirani, \& Pirie, 2011).

The corneal tissue can be completely restored only with the use of donor transplants. Their preparation, storage and application are complicated by a number of factors, which constantly attracts the researchers' attention. Firstly, the graft preparation is associated with a number of legal and physical issues. Secondly, the preservation of biomechanical and optical functions of the graft and its storage are also problems of high complexity (Costa, Leiva, \& Gimenez, 2018; Ito, Terakado, Ichikawa, Zama, Minami, Kudo, \& Kanemaki, 2014; Lacerda, Gimenez, Laguna, \& Costa, 2017; Maggs, Miller, \& Ofri, 2008; Alkan et al, 2017).

A fresh specimen ensures the conservation of all eye functions, so its unavailability in urgent situations, its high antigenicity and risk of immune response are the major drawbacks of that kind of surgery (Laguna, Leiva, Costa, Lacerda, \& Gimenez, 2014; Makra, Biksi, \& Boro, 2013; Townsend, Rankin, Stiles, \& Krohne, 2008).

In world practice, the cryopreserved corneas are defrosted just before application, which facilitates their storage. However, deep freezing damages the endothelial layer, which results in corneal opacity after surgery (Costa, Leiva, \& Gimenez, 2018).

The authors believe that the best results are achieved by means of dehydration over silica gel which allows to preserve low bacterial content for months and low autoimmune response after the transplantation. The corneal opacity due to endothelial dysfunction after surgery is the major disadvantage of this method.

Foreign physicians often prefer fresh or frozen donor corneas. In our routine clinical practice, we use transplants dehydrated over silica gel that helps prevent bacterial contamination, provides implantation with minor complications, and contributes to the preservation and storage. 
Corneal diseases and trauma occupy a leading place in veterinary ophthalmology. At present, there are numerous methods of conservative treatment of corneal diseases, such as combined antibiotic therapy (Shilkin, Kopenkin, Oleinik, \& Smirnova, 2006), novel drugs for corneal cells regeneration, plasma enriched with platelets, autoserum (Shilkin \& Kopenkin, 1999), physiotherapy (Maggs, Miller, \& Ofri, 2013), etc. However, the cases of severe corneal damage that can lead to the loss of an eye and significantly worsen the life of the animal, are common in clinical practice (Shilkin, 2004). In modern world, domestic animals play an important social and often commercial role in human life. The loss of the eyesight or the whole eye significantly worsens the quality of life of a pet and becomes an urgent issue for the pet owner. In such cases, depending on the character and severity of the disease, penetrating keratoplasty can be used for the treatment (Pavlova, 2013; Al-Sharif, 2016).

The aim of the study was to describe the technique of penetrating keratoplasty with preserved corneal grafts and to evaluate the short-term and long-term outcomes.

\section{Material and Methods}

37 animals ( 25 dogs and 12 cats) were subjected to 43 penetrating keratoplasty surgeries. The age of animals varied from 3 months to 14 years.

The biomicroscopy of the anterior segment, tonometry and fluorescent eye test allowed to detect in animals such diseases as corneal ulcers, descemetocoele, corneal perforation and corneal sequestrum.

In all the cases, the authors used corneal grafts dehydrated over silica gel and the ophthalmic microscope Leica M220 F12.

\section{Surgery Description}

The authors used a combined anesthetic support including premedication, systemic and topical anesthesia, induction and inhalation anesthesia. An eyelid retractor was used to dilate palpebral fissure. A Flieringa ring was sutured concentrically to the scleral sulcus to avoid abnormal fluctuation in intraocular pressure and to prevent the vitreous body from prolapse during the surgery. The dehydrated donor cornea was rehydrated in a $1 \%$ solution of gentamicin and $0.9 \%$ saline solution in the ratio of 1 to 5 during 15 minutes. The rehydration during more than 15 minutes leads to the swelling of the graft, which makes it unsuitable for the surgery (Pavlova, Oleinik, Rotanov, Goncharov, \& Lutsay, 2011). The zone of transplantation was limited by corneal trephine. The trephine was chosen so that it would cover the whole area of the damaged tissues. A trephine of corresponding diameter on a special sterile pad made of medical rubber was used to cut a transplant from the donor cornea. This instrument provides for a proper graft alignment on the patient's cornea. In some cases, when a non-standard shape of a transplant was required, a diamond microsurgical knife and micro scissors were used. With the help of a $1.2 \mathrm{~mm}$ knife, the authors performed paracentesis that was used to introduce viscoelastic for the prevention of iris damage and maintenance of the depth of the anterior chamber to exclude hypotonia and thus, a possible hemorrhage. Incision of the cornea was performed with a blunt diamond micro blade "Sakha". This method allowed the surgeons to avoid the iris or lens damage, and to ensure the lubricity and smoothness of the border of the dissected tissues which helped reduce the healing time (Pavlova, 2013). A transparent disk placed in the corneal passage of the recipient was fixed with retention sutures by an atraumatic needle and polyamide thread USP $7 / 0$. The needle was inserted $1 \mathrm{~mm}$ from the wound border via the cornea without penetration into the internal endothelial layer. Four sutures were placed for 3, 6, 9 and 12 hours, respectively. Meridians layout helped the surgeons to position the transplant properly. Further, 4-8-12 single knot fixation sutures were placed depending on the diameter of the transplant at the distance 1$1.5 \mathrm{~mm}$ from each other (Figure 1). A solution of Mesatonum was injected into the anterior chamber for the mydriasis effect to prevent adhesions between the iris and cornea (Figure 2). At the end of the surgery, the Flieringa ring and eyelid retractor were removed and the eye fissure was closed by suturing the eyelids for 2 weeks (blepharorrhaphy). During the postoperative period, antibiotic therapy (local and systemic), mydriatics for the prevention of synechias and drugs for the stimulation of cornea regeneration were indicated for 7 days. 10-14 days after the surgery, the outer sutures were removed. The corneal sutures were removed on the 21 st-30th day under sedation.

Table 1. Types of complications

\begin{tabular}{lll}
\hline Groups and types of complications & & Quantity \\
\hline \multirow{2}{*}{ Complications associated with the poor fixation of the transplant } & Sutures failure & $1(2.326 \%)$ \\
& Anterior synechias & $1(2.326 \%)$ \\
Transplant alterations & Erosion & $1(2.326 \%)$ \\
Other & Transplant disease & $1(2.326 \%)$ \\
& Secondary glaucoma & $1(2.326 \%)$
\end{tabular}




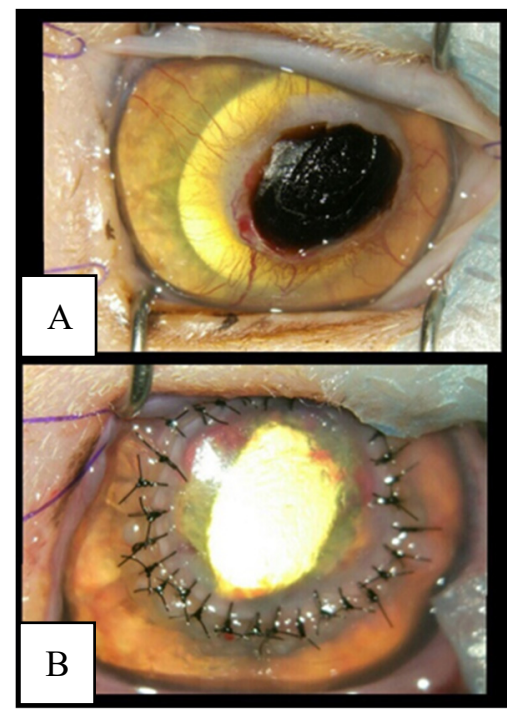

Figure 1.

A. Corneal sequestrum in a cat;

B. Subtotal keratoplasty

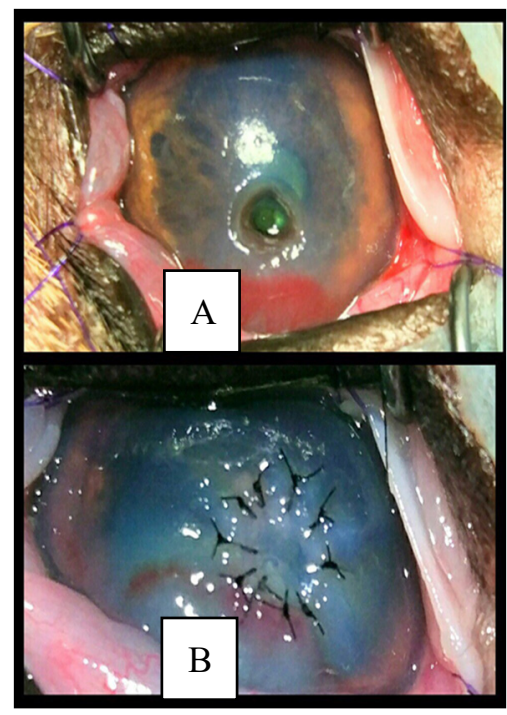

Figure 2.

A. Descemetocele in a Cane Corso dog;

B. Mydriasis after the injection of Mesatonum solution into the anterior chamber

\section{Results}

A favorable outcome was observed in 37 eyes (88.37\%). The cornea was replaced, in cases of corneal perforation the anterior chamber was formed, the sequesters were dissected completely. Transplant survival was complete. Visual function was restored partly or completely. The complications were observed in 5 eyes (11.63\%) (Table 1).

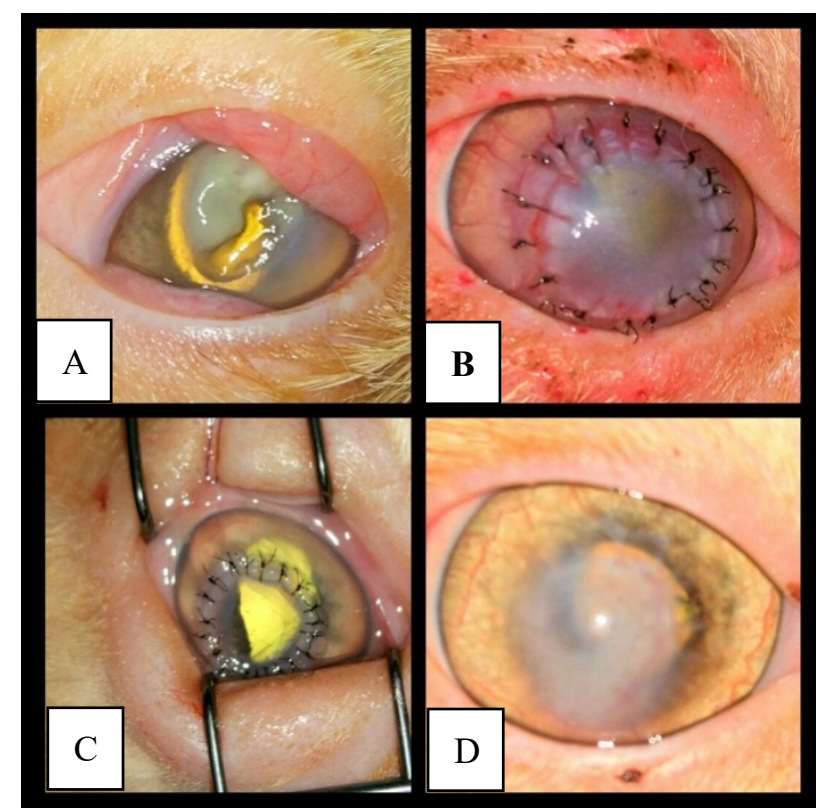

Figure 3. A. Septic ulcer of the cornea in a mixed breed cat; B. Penetrating keratoplasty; C. 14 days after the surgery; D. 3 months after the surgery

Complications associated with transplant rejection were caused by suture failure $(2.326 \%)$ and anterior synechia in 1 eye $(2.326 \%)$. In the case of suture failure, resuture was performed, which led to a complete transplant retention. Anterior synechias were not dissected because of the high risk of intraocular hemorrhage that occurs 
after the injury of the iris. Complications associated with the defect of the transplant were corneal erosion in 1 eye $(2.326 \%)$ and disease of the transplant in 1 eye $(2.326 \%)$. Both pathologies were treated by therapy. Secondary glaucoma developed in the late postoperative period ( 3 months later) on 1 eye $(2.326 \%)$. Hypotensive medication having failed, the animal underwent eyeball endoprosthetics.

Long-term observation of animals (during more than 12 weeks) showed a partial reduction of transplantation area (Figure 3 ) in 11 cats $(25.58 \%)$. In 9 brachycephalic dogs $(20.93 \%)$, the pigmentation occurred in the transplantation zone.

\section{Discussion}

The cornea damage is fourth among the causes of vision loss (after cataract, glaucoma and age-related degeneration of the macula).

Corneal defects often occur under the influence of foreign object penetration or abrasion. In case of minor traumas (the epithelial basement membrane is untouched), the injury is cured in a few days without complications. Deeper damages healing can take up to 2 months. The epithelium recovery occurs due to the following consecutive processes:

- migration of epithelial cells (if the basement membrane is not damaged, the wound is closed due to the migration of neighboring cells);

- mitosis (division of cells) - neighboring cells having completely closed the epithelium defect, their proliferation begins;

- at the final stage the cells adhere to neighboring ones and the vinculin synthesis increases.

Four factors are important for normal epithelium regeneration: undamaged basement membrane, sufficient amount of vitamin A, normal tear film and undamaged sensory nerves.

Deeper damage is characterized by the following processes:

- synthesized fibrin covers the damaged area;

- edema of layers adjacent to the wound develops;

- after a few hours, polymorphonuclear leukocytes and proteolytic enzymes appear in the damaged area;

- synthesis of DNA increases in the keratocytes;

- in 3 days, keratocytes and collagen fibers appear in wound edges.

Newly synthesized collagen fibers are located chaotically, which decreases the transparency of the cornea in the area of their formation. Cicatricial tissue appears in the damaged area approximately in 6 months.

Therapeutic methods of cornea treatment are not effective because they do not solve the problem of epithelial cells adhesion. Surgery aimed at removing the outer layer the cornea including the area of the epithelial basement membrane and the superficial part of the stroma is much more effective as the wound is healed due to the complete regeneration of anterior limiting membrane, and not only the epithelial layer.

The aim of the performed study was to describe the technique of the penetrating keratoplasty with a preserved donor graft in the early and late postoperative periods.

During the study 37 animals were diagnosed with the corneal ulcer, descemetocoele, corneal perforation and corneal sequestrum with the help of biomicroscopy of the anterior section, tonometry, and fluorescent eye test. 43 animals underwent penetrating keratoplasty with the donor cornea dehydrated over silica gel. The graft was rehydrated, the paracentesis was performed in order to introduce viscoelastic that helps prevent iris trauma, maintain the proper depth of the anterior chamber to avoid hypotonia and possible hemorrhage. The final dissection was performed with a blunt diamond blade "Sakha" which allowed the surgeon to avoid iris or lens trauma. The solution of Mesatonum was injected into the anterior chamber to obtain the mydriatic effect and prevent adhesions between iris and cornea. In 10-14 days, outer sutures were removed.

Corneal transplantation results in a significant improvement of the eyesight in animals with cornea diseases and contributes to improve eyesight in case of eye injuries. Usually, the surgery is performed under general anesthesia. The cornea is a relatively simple tissue that does not contain blood vessels, so most of cornea transplant procedures are successful. At the first stage of post-surgery care, antimicrobial therapy and glucocorticoids are indicated to prevent possible transplant rejection. In a few months after the transplantation, the sutures are removed. However, intensive physical efforts should be avoided for some years.

The corneal transplantation involves the replacement of the damaged tissue with the tissue obtained from a donor. 
There are two types of transplantation approaches: total replacement of cornea (penetrating keratoplasty (PK)) and removing the inner lining of the cornea (endothelial keratoplasty (EK)).

The damaged central part of the cornea is replaced with a transplant provided by a local eye tissue bank. The cornea damaged by a disease causes the worsening of the eyesight because it cannot provide proper refractive ability. Transplantation of the cornea can solve the problem of improving the eyesight.

The surgeons commonly use fresh or frozen donated tissue in clinical practice. The present study showed that the best results have been achieved with the transplant dehydrated over silica gel. The method of conservation is simple and the material is easy to store and keeps bacterial content low for months. This method provides an adequate autoimmune response after the transplantation.

During the preservation and dehydration over silica gel, the donor cornea loses its clearance because of corneal endothelium damages. However, in cases with vast total damage of the cornea, it is impossible to perform corneo conjunctival transposition, and replacement of damaged part with a conjunctival patch results in a nontransparent and thick scarring. To reduce the immune response in the postoperative period, corticosteroids were indicated in a number of cases, which contributed to reduce the inflammation.

A favorable surgeries outcome was observed in $88.37 \%$ of cases. The corneal integrity was restored, the anterior chamber was formed, and sequestrum was dissected completely in cases of corneal perforation. The transplants did not fail and visual functions were partly or completely preserved. The complications were observed in $11.63 \%$ of cases.

\section{Conclusions}

1. Therapeutic approaches for corneal defects are ineffective because they do not solve the problem of epithelial cells adhesion. Surgery is much more effective in this case.

2. Penetrating keratoplasty is aimed at the replacement of damaged cornea with donor corneal graft. The transplantation provides the improvement of the eyesight in animals with corneal diseases and eyesight recovery in animals with eye injuries.

3. The outcomes of corneal transplantation using the proposed technique were excellent in $88.37 \%$ of cases. Thus, it can be recommended in emergency treatment of animal cornea.

4. Fresh or frozen donor material is often used in clinical practice. However, the present study showed that the best results are achieved with donor grafts dehydrated over silica gel, which are easy to store and can preserve low bacterial content for months. This method provides an adequate immune response after the transplantation.

5. The donor cornea dehydrated over silica gel is a valuable material for transplantation because it can be easily prepared and stored for a long time, and rehydrated quickly.

6. The present study have shown that dehydrated corneal grafts have a reasonable success rate, therefore, they can be recommended for the interbreed corneal transplantations in animals.

\section{Conflict of interests}

The authors declare that there is no conflict of interests regarding the publication of this paper.

\section{References}

Alkan, A., Mızrak, D., Şenler, F. Ç., \& Utkan, G. (2017). Inadequate Nutritional Status of Hospitalized Cancer Patients. Journal of Clinical and Experimental Investigations, 8(1), 15-21.

Al-Sharif, F. M. (2016). Association between insulin resistance and inflammatory cytokines among obese Saudi type 2 diabetic with vitamin D deficiency. European Journal of General Medicine, 13(4), 91-96.

Brunette, I., Rosolen, S. G., \& Carrier, M. (2011). Comparison of the Pig and Feline Models for Full Thickness Corneal Transplantation. Veterinary Ophthalmology, 14, 365-377.

Costa, D., Leiva, M., \& Gimenez, T. P. (2018). Cryopreservation $\left(-20{ }^{\circ} \mathrm{C}\right)$ of Canine Corneoscleral Tissue: Histological, Microbiological, and Ultrastructural Study. Veterinary Ophthalmology, 18, 291-296.

Gelatt, K. N., Gilger, B. C., \& Kern, T. J. (2013). Veterinary Ophthalmology (5th ed., p. 2184). Ames: WileyBlackwell.

Ito, S., Terakado, K., Ichikawa, Y., Zama, T., Minami, T., Kudo, S., \& Kanemaki, N. (2014). Repair of a Traumatic Corneal Laceration in a Cat Using a Tectonic Heterograft. e-Polish Journal of Veterinary Ophthalmology, 2, $1-6$. 
Lacerda, R., Gimenez, T. P., Laguna, F., \& Costa, D. (2017). Corneal Grafting for the Treatment of Full-Thickness Corneal Defects in Dogs: A Review of 50 Cases. Veterinary Ophthalmology, 20, 222-231.

Laguna, F., Leiva, M., Costa, D., Lacerda, R., \& Gimenez, T. P. (2014). Corneal Grafting for the Treatment of Feline Corneal Sequestrum: A Retrospective Study of 18 Eyes (13 Cats). Veterinary Ophthalmology, 18, 291296.

Maggs, D. J., Miller, P. E., \& Ofri, R. (2008). Slatter's Fundamentals of Veterinary Ophthalmology (4th ed., p. 482). St. Louis: Saunders Elsevier.

Maggs, D. J., Miller, P. E., \& Ofri, R. (2013). Slatter's Fundamentals of Veterinary Ophthalmology (p. 506). St. Louis: Saunders.

Makra, Z., Biksi, I., \& Boro, G. (2013). Penetrating Keratoplasty for Treatment of Corneal Abscesses in HorsesCase Report. Acta vet BRNO, 82(3), 303-307.

Pavlova, T. N. (2013). Keratoplasty in Veterinary Ophthalmology (p. 155) (Ph.D. Thesis). Moscow.

Pavlova, T. N., Oleinik, V. V., Rotanov, D. A., Goncharov, D. V., \& Lutsay, V. I. (2011). Benefits and Drawbacks of different corneal transplants used in Modern Veterinary Ophthalmology. In Collection of Theses of II AllRussian Conference on Veterinary Surgery (pp. 125-129). Moscow.

Pumphrey, S. A., Pizzirani, S., \& Pirie, C. G. (2011). 360-Degree Conjunctival Grafting for Management of Diffuse Keratomalacia in a Dog. Veterinary Ophthalmology, 14, 209-213.

Shilkin, A. G. (2004). Septic Corneal Ulcers in Dogs and Cats. Veterinary, 5, 53-55.

Shilkin, A. G., \& Kopenkin, E. P. (1999). Corneal Sequester, Etiopathogenesis, Diagnostics and Treatment. Veterinary Doctor, 7-8, 34-36.

Shilkin, A. G., Kopenkin, E. P., Oleinik, V. V., \& Smirnova, S. V. (2006). Comparative Efficiency of Different Forms of Fluoroquinolones in Veterinary Ophthalmology. In Materials of Moscow international Veterinary Congress (pp. 158-161).

Townsend, W. M., Rankin, A. G., Stiles, J., \& Krohne, S. G. (2008). Heterologous Penetrating Keratoplasty for Treatment of a Corneal Sequestrum in a Cat. Veterinary Ophthalmology, 11, 273-278.

\section{Copyrights}

Copyright for this article is retained by the author(s), with first publication rights granted to the journal.

This is an open-access article distributed under the terms and conditions of the Creative Commons Attribution license (http://creativecommons.org/licenses/by/4.0/). 